Annts Limnol. 17 (2) 1981 . 121-141.

\title{
CONTRIBUTION A LA CONNAISSANCE DES OLIGOCHETES AQUATIQUES DU BASSIN DE L'ARGENS (VAR, FRANCE)
}

\author{
par N. GIANI ${ }^{1}$ et E. MARTINEZ-ANSEMIL ${ }^{2}$
}

\begin{abstract}
Ce travail est une contribution à la connaissance faunistique et écologique des oligochètes de deux cours d'eau du Midi de la France: l'Argens et un de ses affluents, l'Eau Salée. Nous présentons une première diagnose d'un Tubificidae indéterminé du genre Peloscolex et nous donnons un complément à la description d'Epirodrilus pygmaeus Hrabe qui est signalé ici pour la première fois en France. Nous abordons également quelques points intéressants concernant la répartition géographique de certaines espèces: Chaetogaster setosus Svetlov, Tubifex tubifex blanchardi Vejdovsky, Epirodrilus pygmaeus Hrabe et Lumbricillus kaloensis Nielsen et Christensen.

Les périodes de maturité observées pour les oligochètes de ces cours d'eau sont récapitulées dans un tableau. A partir de nos observations et des données de la littérature nous précisons les préférendums écologiques de chacune des principales espèces avant de conclure sur la répartition longitudinale des oligochètes dans ces deux cours d'eau.
\end{abstract}

\section{The aquatic oligochaetes of the Argens basin [Var, France].}

This work is a contribution to the faunistic and ecological knowledge of oligochaetes in two rivers of Southern France: the Argens and one of its tributaries, the Eau Salée. We present a first description of an indeterminate tubificid of the genus Peloscolex, and a complement to the description of Epirodrilus pygmaeus Hrabe which is recorded here for the first time in France. We also deal with several interesting points concerning the geographical distribution of certain species: Chaetogaster setosus Svetlov, Tubifex tubifex blanchardi Vejdovsky, Epirodrilus pygmaeus Hrabe, and Lumbricillus kaloensis Nielsen \& Christensen.

The periods of maturity for the oligochaetes of these rivers are summarised in a table. From our own study and literature data, we define the ecological requirements of each of the principal species before making general conclusions on the longitudinal distribution of oligochaetes in the two rivers.

Lors d'une étude sur la répartition des principaux groupes de macroinvertébrés benthiques dans la rivière Argens (Martinez-Ansemil 1978), nous avons eu l'occasion de récolter un grand nombre d'Oligochètes que nous examinons dans le présent travail. Aux oligochètes de l'Argens, nous avons adjoint ceux de l'Eau Salée, un de ses principaux

1. Laboratoire d'Hydrobiologie, ERA 702, Université Paul-Sabatier, 118, route de Narbonne, 31062 Toulouse Cedex.

2. Colegio Universitario de Orense, C. General Franco, 35 Orense (Espagne). 
affluents, qui nous ont été aimablement légués par notre collègue B. Dehzad.

Ce travail est surtout consacré à une étude faunistique globale (taxonomie, répartition et période de maturité) et autoécologique des principales espèces. Nous avons également envisagé la répartition longitudinale de ce groupe d'invertébrés dans les deux cours d'eau précédemment cités.

\section{1. - MILIEUX ET METHODES}

Le bassin versant de l'Argens (fig. 1) est situé entièrement dans le département du Var (Midi de la France). Du point de vue géologique, il est essentiellement formé par des terrains calcaires du trias et du jurassique; de par sa situation il subit l'influence d'un climat méditerranéen. La plus grande partie de son réseau hydrographique est, par conséquent, soumise à de brusques variations de débit.

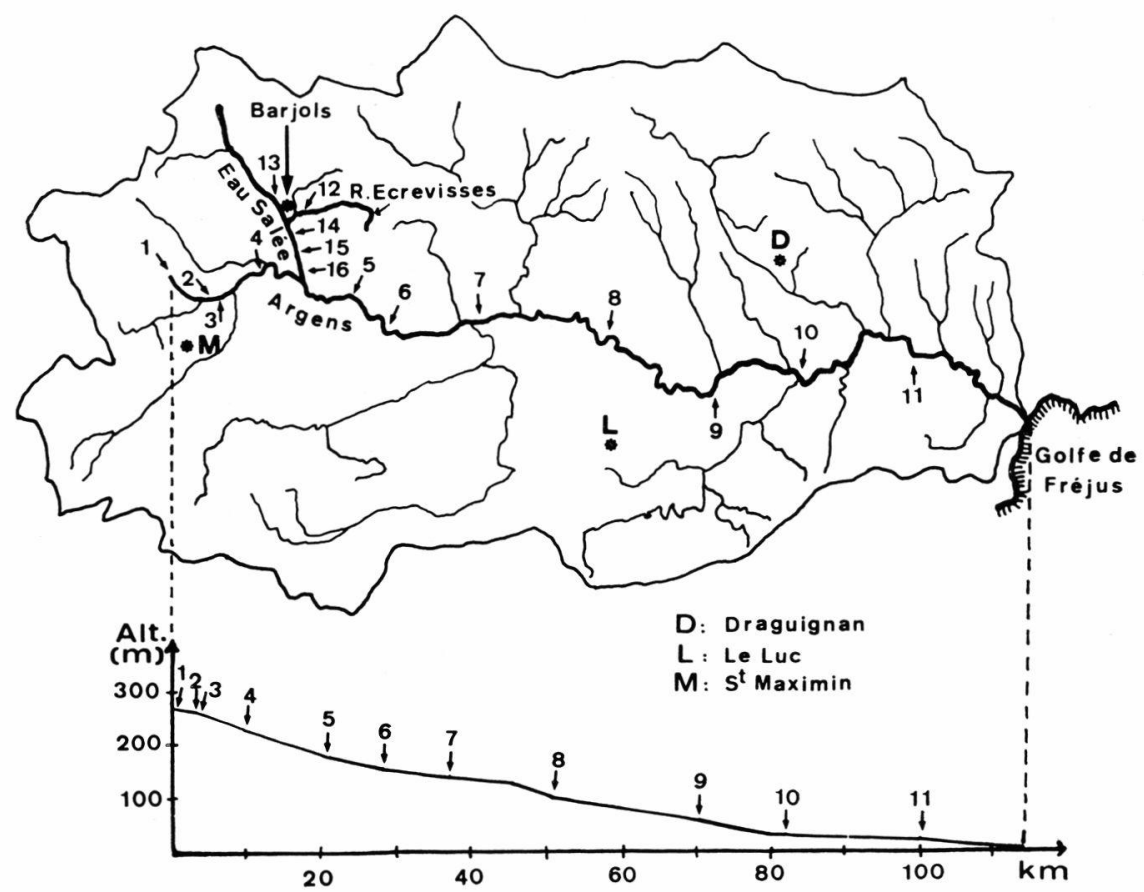

Fig. 1. - Bassin versant de l'Argens : réseau hydrographique, profil longitudinal et localisation des stations d'étude.

Nous avons retenu 11 stations d'échantillonnage réparties tout le long du cours d'eau principal, l'Argens et 5 situées sur un de ses prin- 
TableaU I. - Principales caractéristiques abiotiques des stations de l'Argens. A : algues filamenteuses, B : blocs, C : cailloux, G : galets, $\mathrm{Gr}$ : graviers, $\mathrm{H}$ : hépatiques, $\mathrm{M}:$ mousses, $\mathrm{S}$ : sables, $\mathrm{T}:$ tuff, $\mathrm{V}:$ vase, $\mathrm{Vp}$ : végétation en faciès lénitique. (1) Les substrats ont été classés selon l'échelle de Cailleux (1954). (2) $\mathrm{La}$ concentration en $\mathrm{Ca}^{++}$varie entre 84 et $133 \mathrm{mg} / \mathrm{l}$ et celle en $\mathrm{Mg}^{++}$entre 8 et $31 \mathrm{mg} / \mathrm{l}$.

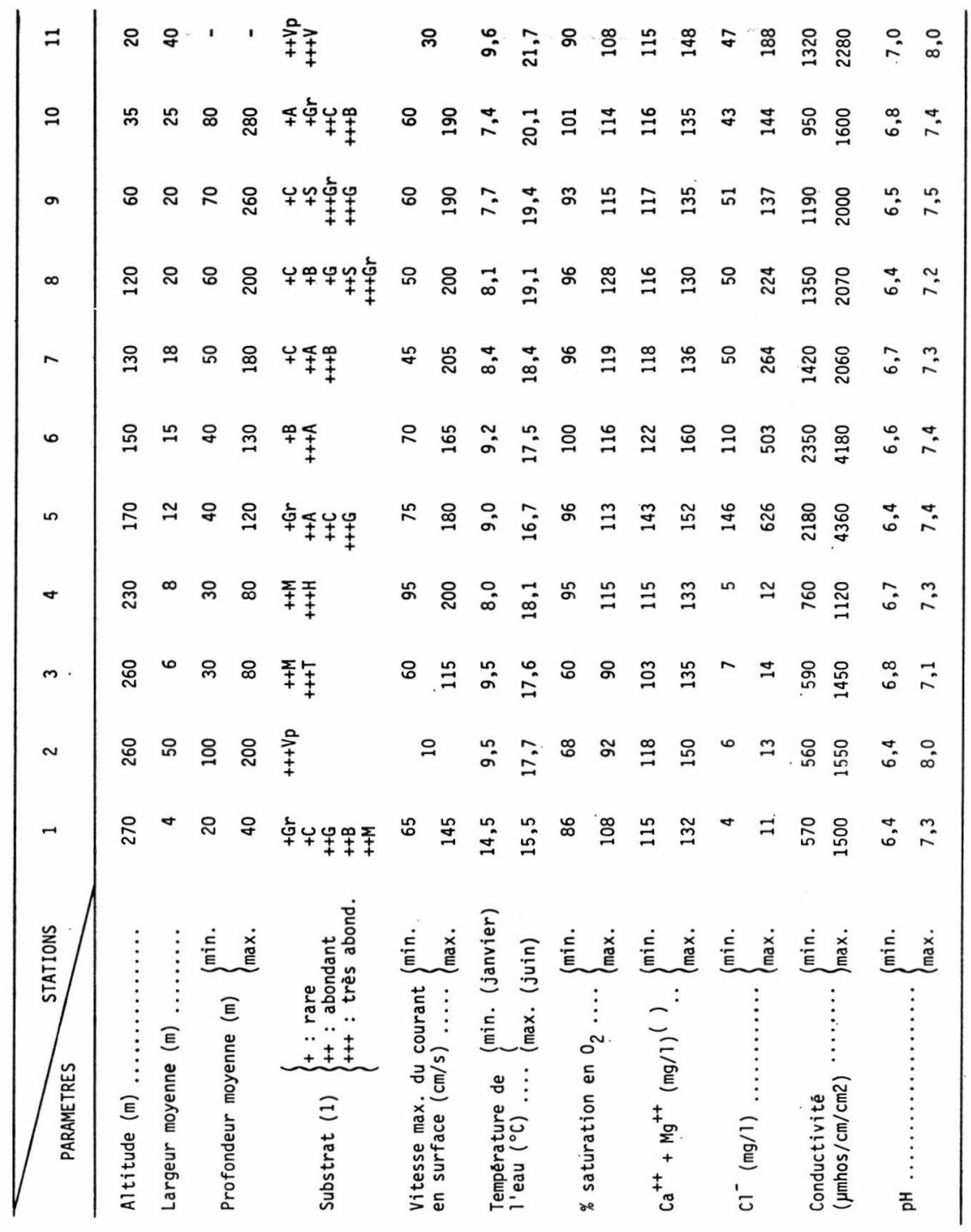

cipaux affluents l'Eau Salée (fig. 1). Dans chacune de ces 16 stations, des prélèvements de faune et des mesures des principaux paramètres physico-chimiques ont été réalisés tout le long de l'année 1976, aux mois de janvier, mars, avril, mai, juin, septembre et novembre dans 
l'Eau Salée et chaque mois dans l'Argens, à l'exception d'octobre en raison d'une crue très importante.

Aux stations 2 et 11 (faciès lénitique) l'échantillonnage de la faune a été réalisé uniquement dans la végétation au moyen d'un filet troubleau de $0,3 \mathrm{~mm}$ de vide de maille, alors que dans toutes les autres stations il a été effectué toujours en faciès lotique (vitesse de courant $>25 \mathrm{~cm} / \mathrm{s}$ ) à l'aide d'un filet de Surber (surface : $1 / 40 \mathrm{~m}^{2}$; vide de maille : $0,3 \mathrm{~mm}$ ).

Lors de chaque prélèvement de faune nous avons noté la vitesse de courant et le type de substrat existant à l'endroit précis ou celui-ci était effectué. Compte tenu de nos méthodes les résultats numériques ont une valeur semi-quantitative pour l'Argens et seulement qualitative pour l'Eau Salée.

Les principales caractéristiques abiotiques des stations sont résumées dans les tableaux I et II. A la vue de ces deux tableaux il convient de souligner les points suivants:

Tableau II. - Principales caractéristiques abiotiques des stations de l'Eau Salée d'après les données de Dehzad (1978). Les valeurs des paramètres chimiques correspondent aux moyennes saisonnières. Les vitesses de courant ont été classées selon l'échelle de Berg (1948). A, B, C, G, Gr, H, M, S : même signification que pour le tableau I.

\begin{tabular}{|c|c|c|c|c|c|}
\hline PARAMETRES STATIONS & 12 & 13 & 14 & 15 & 16 \\
\hline Altitude (m) & 301 & 239 & 215 & 205 & 190 \\
\hline Largeur moyenne $(\mathrm{m})$ & 4 & 6 & 7 & 5 & 10 \\
\hline Profondeur moyenne $(\mathrm{cm})\left(\begin{array}{l}\min . \\
\max .\end{array}\right.$ & $\begin{array}{r}7 \\
25\end{array}$ & $\begin{array}{l}10 \\
30\end{array}$ & $\begin{array}{l}12 \\
35\end{array}$ & $\begin{array}{l}20 \\
50\end{array}$ & $\begin{array}{l}10 \\
35\end{array}$ \\
\hline Substrat $\left\{\begin{array}{l}+ \text { rare } \\
++ \text { abondant } \\
+++ \text { très abondant }\end{array}\right.$ & $\begin{array}{r}+B \\
+++H\end{array}$ & $\begin{array}{r}+C \\
+A \\
++G r \\
++S\end{array}$ & $\begin{array}{r}++B \\
+++M\end{array}$ & $\begin{array}{r}+B \\
+G r \\
+S \\
+M \\
+A \\
++C\end{array}$ & $\begin{array}{r}+S \\
+M \\
++C \\
+++G\end{array}$ \\
\hline $\begin{array}{l}\text { Vitesse maximale du } \\
\text { courant en surface } \ldots .\left(\begin{array}{l}\min . \\
(\mathrm{cm} / \mathrm{s})(1)\end{array}\right.\end{array}$ & $\begin{array}{l}\text { modérée } \\
\text { rapide }\end{array}$ & $\begin{array}{l}\text { lente } \\
\text { rapide }\end{array}$ & $\begin{array}{l}\text { lente } \\
\text { rapide }\end{array}$ & $\begin{array}{l}\text { lente } \\
\text { rapide }\end{array}$ & $\begin{array}{l}\text { lente } \\
\text { rapide }\end{array}$ \\
\hline Température de $l^{\prime}$ eau $\left({ }^{\circ} \mathrm{C}\right) \int_{\max }^{\min .}$. & $\begin{array}{l}\text { 8/janv. } \\
16 / \text { juin }\end{array}$ & $\begin{array}{r}6,5 / \text { janv } \\
15,2 / \text { sept }\end{array}$ & $\begin{array}{l}7,9 / \text { janv. } \\
15,5 / \text { sept. }\end{array}$ & $\begin{array}{l}6,9 / \text { janv } \\
16,1 / \text { mai }\end{array}$ & $\begin{array}{l}\text { 5,5/janv. } \\
\text { 16/sept. }\end{array}$ \\
\hline$\%$ saturation en $\mathrm{O}_{2} \ldots .\left(\min _{\max }\right.$. & $\begin{array}{l}106 \\
114\end{array}$ & $\begin{array}{r}92 \\
108\end{array}$ & $\begin{array}{l}85 \\
96\end{array}$ & $\begin{array}{r}90 \\
100\end{array}$ & $\begin{array}{r}95 \\
104\end{array}$ \\
\hline $\mathrm{Ca}^{++}(\mathrm{mg} / 1) \ldots$ & $\begin{array}{r}84 \\
100\end{array}$ & $\begin{array}{l}121 \\
172\end{array}$ & $\begin{array}{r}93 \\
132\end{array}$ & $\begin{array}{r}71 \\
128\end{array}$ & $\begin{array}{r}85 \\
136\end{array}$ \\
\hline $\mathrm{Mg}^{++}(\mathrm{mg} / 1) \ldots$ & $\begin{array}{l}21 \\
26\end{array}$ & $\begin{array}{l}32 \\
49\end{array}$ & $\begin{array}{l}24 \\
36\end{array}$ & $\begin{array}{l}30 \\
34\end{array}$ & $\begin{array}{l}10 \\
33\end{array}$ \\
\hline $\mathrm{Cl}^{-}(\mathrm{mg} / 1) \ldots$ & $\overline{-}$ & $\begin{array}{r}400 \\
2200\end{array}$ & $\begin{array}{r}190 \\
1020\end{array}$ & $\begin{array}{l}205 \\
980\end{array}$ & $\begin{array}{l}155 \\
930\end{array}$ \\
\hline $\mathrm{pH}$ & \multicolumn{5}{|c|}{ En $n_{1}$ e 6,6 et 7,6} \\
\hline
\end{tabular}


a) la concentration en chlorures très élevée des eaux de l'Eau Salée (150 à $2200 \mathrm{mg} / \mathrm{l} \mathrm{de} \mathrm{Cl}^{-}$). Selon Nisbet et Verneaux (1970), au-delà de $100 \mathrm{mg} / \mathrm{l}$ (classe 7) on a affaire à des cours d'eau très particuliers ou fortement pollués.

b) la forte minéralisation des eaux de l'Argens, avec une dureté totale très élevée $\left(\mathrm{C}^{++}+\mathrm{Mg}^{++}=103\right.$ à $\left.160 \mathrm{mg} / \mathrm{l}\right)$ - catégorie 6 et 7 dans la classification de Nisbet et Verneaux (1970) - et une teneur en $\mathrm{Cl}^{-}$très importante en aval de la confluence avec l'Eau Salée (4 à $13 \mathrm{mg} / \mathrm{l}$ avant la confluence et 47 à $626 \mathrm{mg} / \mathrm{l}$ après celle-ci).

c) la très grande hétérogénéité dans la composition et la nature du substrat qui recouvre le lit des différents secteurs de ces deux cours d'eau.

d) la singularité des stations 2 et 11 qui représentent des milieux typiquement lénitiques par rapport à toutes les autres stations.

e) les conditions de sténothermie de la station $1\left(15 \pm 0,5{ }^{\circ} \mathrm{C}\right)$ qui est la source de l'Argens, l'eau provenant d'un important réservoir souterrain.

\section{2. - FAUNISTIQUE}

\subsection{Composition spécifique}

Au cours de la présente étude nous avons recensé 43 espèces d'Oligochètes pour un total de 6689 individus : 35 espèces pour 5970 individus dans l'Argens (Tableau III) et 22 espèces pour 719 individus dans l'Eau Salée (Tableau IV). Le manque de prélèvements dans les milieux vaseux est à l'origine du petit nombre de Tubificidae répertoriés dans ces deux cours d'eau.

\subsection{Remarques taxonomiques et biogéographiques}

\subsubsection{COMPLÉmENTS a LA DESCRIPTION d'Epirodrilus pygmaeus (Hrabe 1935)}

Cette espèce a été décrite de Tchécoslovaquie par Hrabe en 1935 mais depuis cette date elle n'a été récoltée qu'une seule fois, en Pologne, par Legezensky (1971). Comme parmi les 14 individus que nous avons récoltés, 9 étaient sexuellement matures ( 3 le 8 mars, 1 le 17 mai et 5 le 15 juin 1976), il nous a paru opportun de compléter la description de cette forme. En effet, la diagnose de Hrabe (1935) est essentiellement basée sur des coupes histologiques et elle manque, selon nous, de dessins d'ensemble de l'appareil génital mâle et de la spermathèque isolée après dissection. Il convient de souligner ici, une fois de plus, que, dans l'éternel débat entre les écologistes et les taxonomistes (voir Brinkhurst 1980), si les coupes histologiques apportent des 
TABLEAU III. - Oligochètes récoltés dans les différentes stations d'échantillonnage de l'Argens: composition spécifique et abondances relatives.

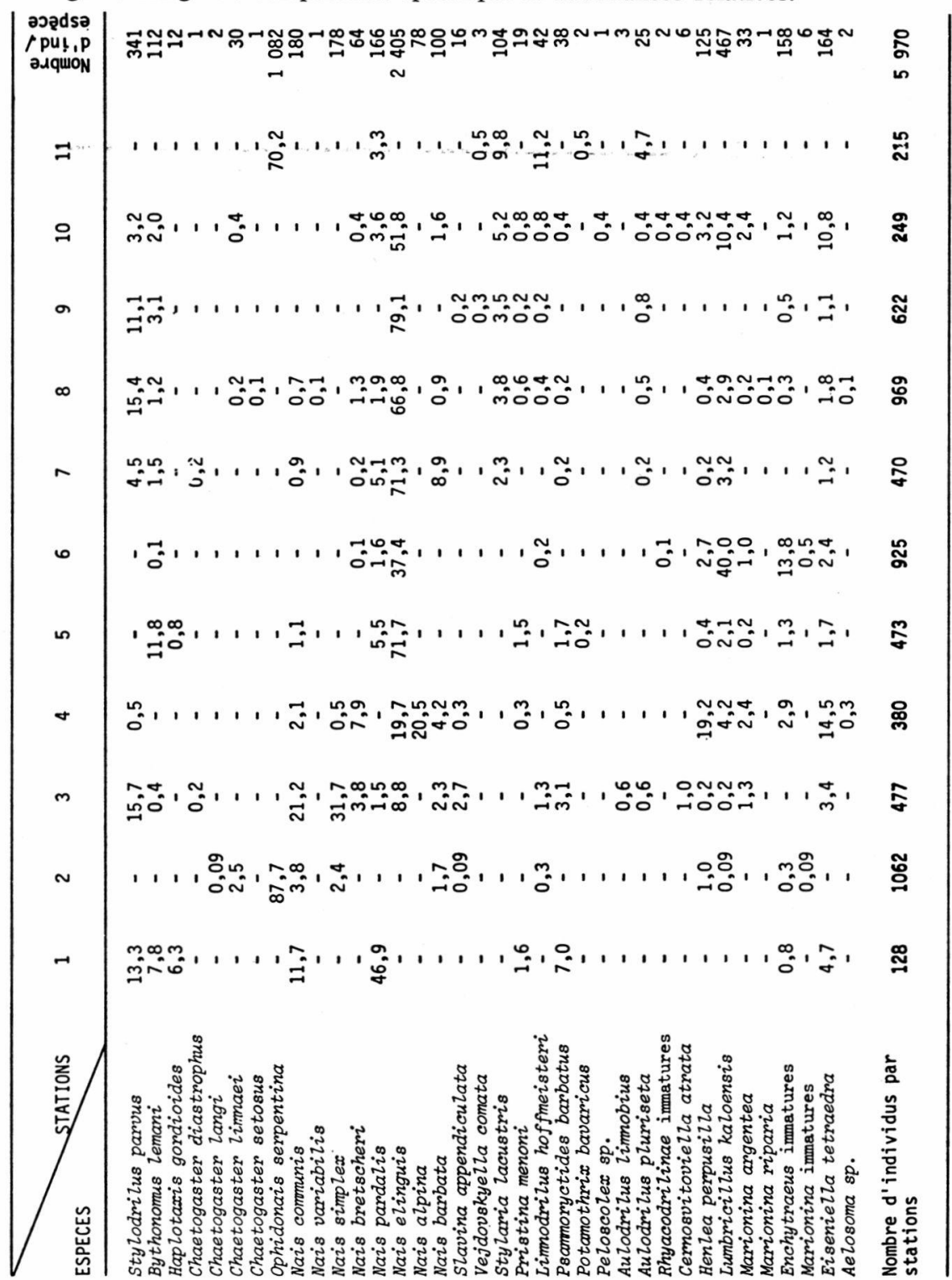

renseignements précieux, elles restent difficiles à mettre en œuvre pour des déterminations multiples; les écologistes préfèrent donc la dissection lorsqu'elle est possible.

Nous limiterons notre description aux seuls points susceptibles de 
compléter ou d'amender la diagnose de Hrabe (1935). Ces points sont regroupés dans le tableau $\mathrm{V}$.
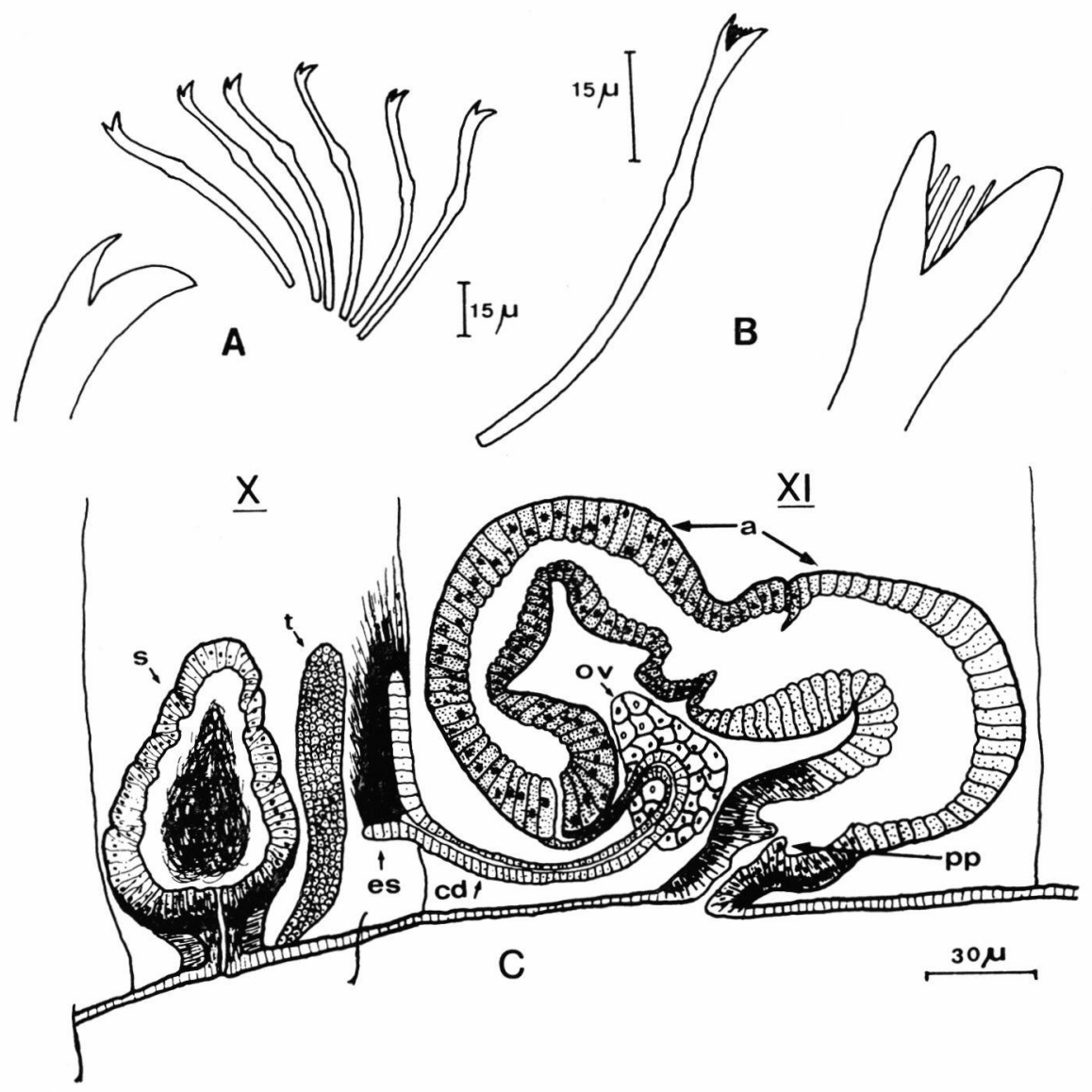

Fig. 2. - Epirodrilus pygmaeus (Hrabe). - A: soies ventrales de la région moyenne ; $\mathrm{B}$ : crochet pectiné dorsal ; $\mathrm{C}$ : appareil génital (d'après dissection) ; a: atrium, cd: canal déférent, es: entonnoir spermatique, ov: ovaire, pp: pseudopénis, $s$ : spermathèque, $t:$ testicule.

\section{Remarques}

Le nombre de segments (55-52) donné par Hrabe nous paraît être probablement entaché d'une erreur typographique : en effet 55-62 segments nous semblerait plus logique.

En ce qui concerne les soies (fig. $2 \mathrm{~A}$ et B), nos observations sont en accord avec la description originale ; toutefois, la différence d'épaisseur, signalée par l'auteur, entre les dents externes des crochets pectinés nous apparaît plus accusée que celle qu'il dessine.

La descripiton de l'appareil génital mâle telle qu'elle a été réalisée 
Tableau V. - Comparaison entre la description d'Epirodrilus pygmaeus selon Hrabe (1935) et nos propres observations.

\begin{tabular}{|c|c|c|c|}
\hline & & NOS OBSERVATIONS & DESCRIPTION DE HRABE \\
\hline Taille ...... & $\left\{\begin{array}{l}\text { Longueur } \\
\text { Nombre de segments }\end{array}\right.$ & $\begin{array}{l}6 \mathrm{~mm} \text { après fixation } \\
60 \text { à } 65\end{array}$ & $\begin{array}{l}15-20 \mathrm{~mm} \text { in vivo } \\
55 \text { à } 52 \text { (voir texte) }\end{array}$ \\
\hline \multirow{3}{*}{$\begin{array}{l}\text { Nombre de } \\
\text { soies } \ldots \ldots . .\end{array}$} & SVentrales & $\begin{array}{l}\text { région antérieure : } 4 \text { à } 6 \\
\text { région postérieure : } 3 \text { à } 4\end{array}$ & $\begin{array}{l}\text { (3) } 4 \text { à } 5 \\
3 \text { à } 5\end{array}$ \\
\hline & $\left\{\begin{array}{l}\text { Dorsales } \\
\text { - Capillaires }\end{array}\right.$ & $\begin{array}{l}\text { région antērieure : }(2)-3-(4) \\
\text { rēgion postêrieure : absentes }\end{array}$ & $2(3)$ \\
\hline & $\{$ - crochets pectinès & $\begin{array}{l}\text { région antérieure : } 3 \text { à } 4(5) \\
\text { région postèrieure : } 2-3\end{array}$ & 3 à 4 \\
\hline \multirow{3}{*}{$\begin{array}{l}\text { Longueur } \\
\text { des soies }\end{array}$} & Sventrales & $\begin{array}{l}\text { région antêrieure : } 65-75 \mu \\
\text { région postérieure : } 45-50 \mu\end{array}$ & \\
\hline & $\left\{\begin{array}{l}\text { Dorsales: } \\
\text { - capillaires }\end{array}\right.$ & $\begin{array}{l}\text { région antérieure : } 240-270 \mu \\
\text { région moyenne : } 120-210 \mu\end{array}$ & \multirow[t]{2}{*}{$\begin{array}{l}6 \text { au segment } \\
\text { correspondant }\end{array}$} \\
\hline & - crochets pectinés & région antérieure : $65-72 \mu$ & \\
\hline \multicolumn{2}{|c|}{ Coelomocytes (diamètre) } & $10-12 \mu$ & \multirow[t]{2}{*}{$15 \mu$} \\
\hline \multicolumn{2}{|c|}{ Glandes pharyngiennes } & en IV-V et VI - réduites en VI & \\
\hline \multicolumn{2}{|c|}{ Canal déférent $\left\{\begin{array}{l}\text { Longueur } \\
\text { Diamẽtre externe }\end{array}\right.$} & $\begin{array}{l}60 \mu \\
12 \mu\end{array}$ & ${ }_{13-16}^{200 \mu} \mu$ \\
\hline Atrium $\ldots \ldots$ & $\left\{\begin{array}{l}\text { Longueur } \\
\text { Diamètre }\end{array}\right.$ & $\begin{array}{l}640-700 \mu \\
\text { de } 65 \text { à } 120 \mu \text { selon les zones }\end{array}$ & $\begin{array}{l}430 \mu \\
77 \mu\end{array}$ \\
\hline \multirow{2}{*}{ Spermathèque } & $\left\{\begin{array}{l}\text { Ampoule : } \\
\text { - forme } \\
\text { - longueur } \\
\text { - diamètre }\end{array}\right.$ & $\begin{array}{l}\text { piriforme } \\
190-250 \mu \\
130-180 \mu\end{array}$ & globulaire \\
\hline & $\left\{\begin{array}{l}\text { Canal : } \\
- \text { forme } \\
\text { - diamètre }\end{array}\right.$ & $\begin{array}{l}\text { court et large } \\
60 \mu \text { pour une lumière de } 17 \mu\end{array}$ & court \\
\hline
\end{tabular}

par Hrabe (1935) concorde pleinement avec nos observations; seules les longueurs diffèrent, mais ces variations peuvent simplement résulter de la contraction différente des individus observés. A ce propos nous insisterons, comme l'a fait Hrabe lui-même, sur l'aspect très variable de cet appareil complexe selon son degré de contraction qui peut dépendre du fixateur utilisé. Ainsi, notre dessin (fig. $2 \mathrm{c}$ ) de l'atrium semble correspondre à la forme de ceux fixés au formol dont toute la partie proximale est partout de la même largeur comme le signalait Hrabe (1935, page 7).

\subsubsection{Description de Peloscolex sp.}

Peloscolex sp. diffère de toutes les autres espèces de ce genre actuellement connues en France mais la capture d'un spécimen ne nous a pas permis de réaliser une diagnose complète de cette forme. En attendant de récolter du matériel supplémentaire, nous décrivons ici cet individu capturé à l'état de maturité sexuelle le 19-08-76. 
Longueur : $4 \mathrm{~mm}$ (après fixation). Prostomium et premier segment rétractiles.

Faisceaux dorsaux comportant 1 soie capillaire et 1 soie pectinée (fig. $3 \mathrm{c}$ ). Faisceaux ventraux constitués par 1 ou 2 soies bifides, avec la dent distale nettement plus longue que la proximale dans les segments antérieurs ( $f i g .3 \mathrm{a}$ ) et de la même longueur ou légèrement plus

TABLEAU IV. - Espèces d'Oligochètes récoltées dans les différentes stations d'échantillonnage de l'Eau Salée.

\begin{tabular}{|c|c|c|c|c|c|c|}
\hline Stations & 12 & 13 & 14 & 15 & 16 & $\begin{array}{l}\text { Nombre d' } \\
\text { individus }\end{array}$ \\
\hline Trichodrilus allobrogum & & & & + & & 7 \\
\hline Haplotaxis gordioides & + & & & & & 1 \\
\hline Ophidonais serpentina & & & & + & & 1 \\
\hline Nais communis & + & & & & + & 4 \\
\hline Nais variabilis & + & & & & & 1 \\
\hline Nais simplex & + & & & & & 4 \\
\hline Nais bretscheri & + & & & & & 2 \\
\hline Nais pardalis & + & & & & & 7 \\
\hline Nais elinguis & & + & + & + & + & 109 \\
\hline Nais alpina & + & & & & & 1 \\
\hline Nais pseudobtusa & + & & & & & 1 \\
\hline Pristina menoni & & + & & + & + & 236 \\
\hline Pristina idrensis & + & + & & + & + & 9 \\
\hline Tubifex tubifex tubifex & & & & + & & 1 \\
\hline Tubifex tubifex blanchardi & & & + & + & & 15 \\
\hline Limnodrilus hoffmeisteri & & & + & & + & 3 \\
\hline Limnodrilus udekemanius & & & + & & & 2 \\
\hline Psammoryctides barbatus & + & + & & + & + & 11 \\
\hline Potamothrix bavaricus & & & & & + & 8 \\
\hline Epirodrilus pygmaeus & & + & & + & + & 15 \\
\hline Rhyacodrilus falciformis & + & & & & & 1 \\
\hline Lumbricillus rivalis & + & + & + & + & + & 260 \\
\hline Eiseniella tetraedra & + & + & + & + & + & 20 \\
\hline
\end{tabular}

longue dans les segments postérieurs (fig. $3 \mathrm{~b}$ ). Soies ventrales du segment $X$ modifiées en soies spermathècales ( 1 par faisceau) avec l'extrémité distale excavée en forme de plume. Ces soies se trouvent insérées sur une aire glandulaire ( $f$ ig. $3 \mathrm{~d}$ ). Soies ventrales absentes en XI?

L'appareil génital mâle est formé, de chaque côté, d'un canal déférent long qui débouche dans un atrium recourbé en fer à cheval et dont le diamètre est à peine supérieur à celui du canal déférent. Une prostate est attachée à la partie médiane de la courbure de l'atrium. Le pénis, entouré d'une gaine cuticulaire cylindrique, nettement plus longue que large (fig. $3 \mathrm{e}$ ), rappelle celui de $P$. zavreli Hrabe.

Les spermathèques sont en forme d'ampoule allongée et elles contiennent des spermatophores également allongés.

Cette forme est proche de $P$. speciosus Hrabe et de $P$. zavreli Hrabe 

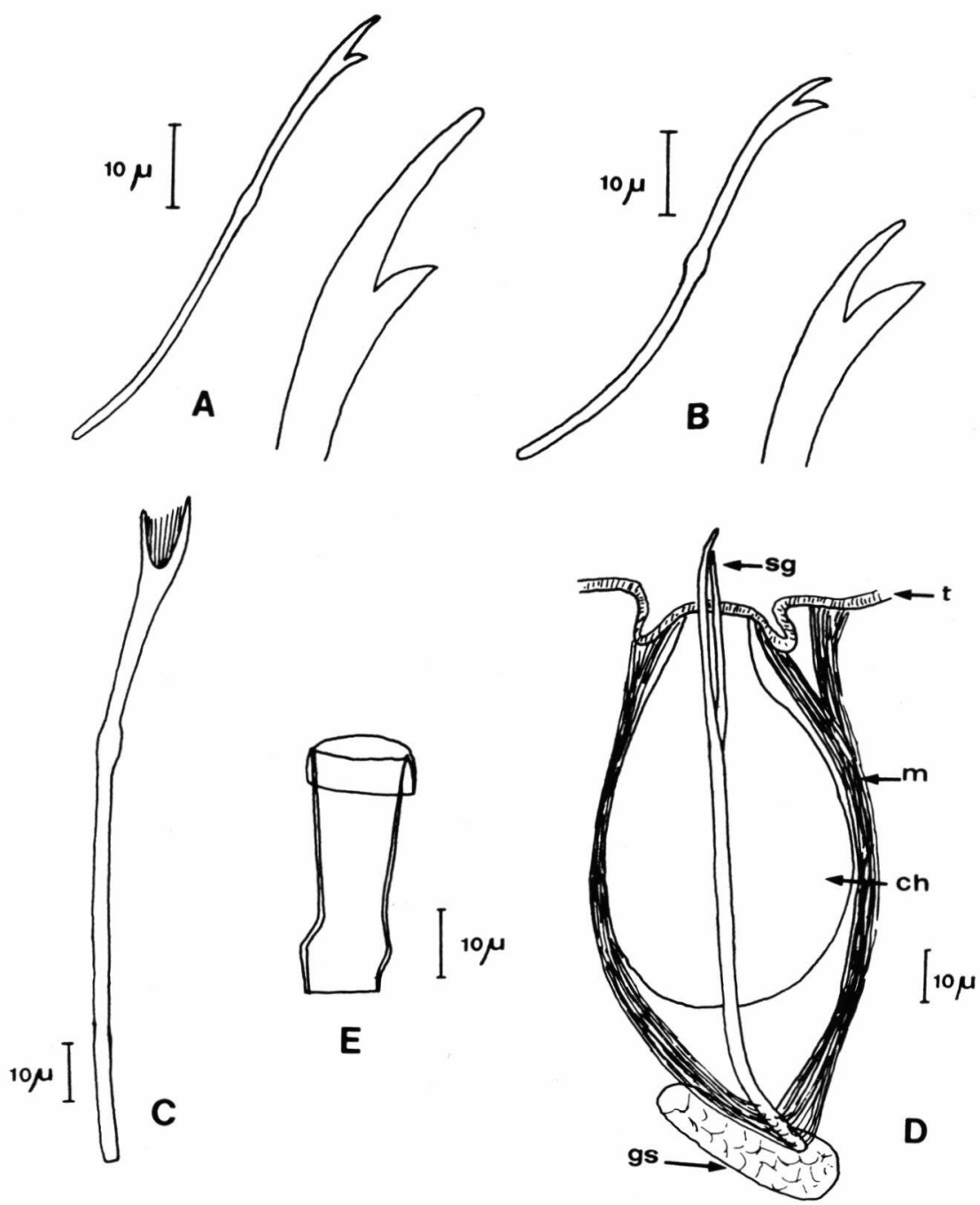

Fig. 3. - Peloscolex $s p$. - A : soie ventrale antérieure; B : soie ventrale postérieure; C : crochet dorsal pectiné ; D : soie spermathécale in situ (ch : chaetophore, gs : glande sétale, $\mathrm{m}$ : muscles, sg : soie, $\mathrm{t}$ : tégument); $\mathrm{E}$ : gaine cuticulaire du pénis.

qui possèdent des soies péniennes en XI alors que nous n'en avons pas observé sur notre spécimen.

\subsubsection{REMARQUES SUR LA DISTRIBUTION DE CERTAINES ESPÈCES}

Chaetogaster setosus. Le seul specimen que nous ayons récolté diffère légèrement de la forme type décrite par Svetlov (1925) et semble appartenir à la variété lemani définie par Juget (1967). En effet, notre 
spécimen possède 7 soies par faisceau sur le segment II et 4 à partir du segment VI comme la variété lemani ( 9 ou 10 en II et de 5 à 8 à partir de VI pour la forme type). Le matériel du Volp (8 individus récoltés par Giani, 1976) que nous avons réexaminé appartient à la forme lemani et un des spécimens ne possède que 6 soies par faisceau sur le segment II. Ces soies ont une longueur de $96 \mu$ maximum et celles des segments VI et postérieurs mesurent de 78 à $93 \mu$ sur l'individu de l'Argens alors que sur ceux récoltés dans le Volp les soies du segment II varient entre 96 et $110 \mu$ et celles du segment VI et suivants entre 81 et $93 \mu$. Pour ces mêmes soies, Juget (1967) a noté des longueurs de 100 à $110 \mu$ et 85 à $100 \mu$ respectivement.

Jusque dans les années 60 C. setosus n'était connu que d'U.R.S.S. ; après la récolte de cette espèce dans le lac Léman Juget (1967) et Wautier et Juget (1969) ont considéré que la variété lemani était une véritable relicte glaciaire. La capture de notre spécimen dans l'Argens (cours d'eau méditerranéen) ainsi que celles dans deux affluents de la Garonne - le Volp à $240 \mathrm{~m}$ d'altitude (Giani 1976) et l'Aveyron à $610 \mathrm{~m}$ (données non publiées) - nous amènent à considérer $C$. setosus var. lemani comme étant la forme occidentale de cette espèce dans son aire de répartition disjointe. En effet, la répartition de cette forme (fig. 4) est très discontinue; elle n'est connue que d'U.R.S.S. : rivière

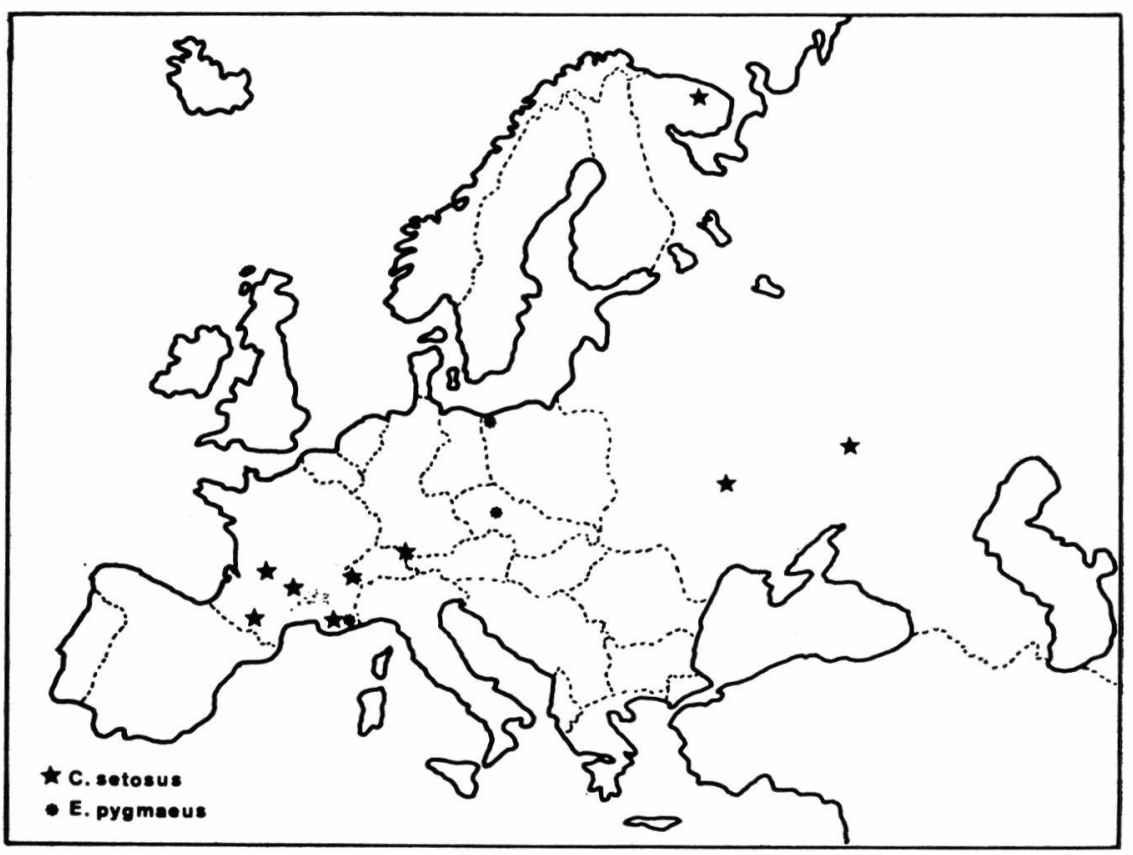

Fic. 4. - Répartition actuelle de Chaetogaster setosus, Svetlov et Epirodrilus pygmaeus (Hrabe) d'après nos données et celles de la littérature. 
Kama (Svetlov 1925), rivière Dniepr (Fomenko 1962), région de Mourmansk (Timm et Popchenko 1978), de France : citations ci-dessus de la Dordogne (Lafont, comm. orale) - et du lac de Constance à la frontière Germano-Suisse (Frenzel 1981).

Tubifex tubifex blanchardi. A notre connaissance la forme blanchardi de $T$. tubifex n'avait jamais été signalée en France. Il s'agit vraisemblablement d'une forme assez rare dans la nature, car depuis sa description par Vejdovsky (1891) en Algérie elle n'a été retrouvée qu'à l'Ile de Corfou (Grèce), en Yougoslavie, Argentine, Paraguay et Birmanie.

Epirodrilus pygmaeus. La présente capture constitue la première citation de ce genre en France. Il semblerait comme le remarquaient Brinkhurst et Jamieson (1971) que le genre Epirodrilus ait une aire de répartition très discontinue dans le monde car sur les 5 espèces décrites 4 ne sont connues que de leur localité type : E. michaelseni Hrabe en Grèce, E. antipodum Cernosvitov au Pérou, E. allansoni Brinkhurst en Afrique du Sud et E. slovenicus Karaman en Yougoslavie. E. pygmaeus n'avait été signalée que deux fois : en Pologne et Tchécoslovaquie (carte de la fig. 4).

Lumbricillus kaloensis. Cette espèce n'a été signalée que très récemment en France (Healy, 1980). Nos récoltes dans l'Argens montrent que la présence de cette forme en Europe peut s'étendre aussi au pourtour de la méditerranée. En effet, outre les captures de Healy (1980) au voisinage de l'Atlantique, cette espèce n'était connue que du Danemark (Nielsen et Christensen 1959), d'Allemagne (Wachs 1967), de Pologne (Kasprzak 1972) et d'Irlande (Mc Grath 1975).

\section{3. - DONNEES BIOLOGIQUES}

\subsection{Périodes de maturité}

Dans le tableau VI, nous avons regroupé les dates auxquelles nous avons capturé des individus matures des principales espèces d'Oligochètes de l'Argens et de l'Eau Salée. A la vue de ce tableau il apparaît que, dans ces deux cours d'eau, c'est en hiver et au printemps qu'un plus grand nombre d'espèces atteignent leur maturité sexuelle. Au contraire, l'été semble être la saison la plus défavorable. Pour les espèces dont la phase de maturité est très étalée tout au long de l'année (S. parvus, B. lemani...) on constate également un minimum estival du nombre des individus matures. 
Tableau VI. - Périodes de captures d'individus matures dans l'Argens (+) et dans l'Eau Salée (o). L'absence de double trait (en haut pour l'Eau Salée, en bas pour l'Argens) indique l'absence de prélèvements à la période considérée. Les flèches correspondent à une augmentation $(\uparrow)$ ou une diminution $(\downarrow)$ des nombres d'individus.

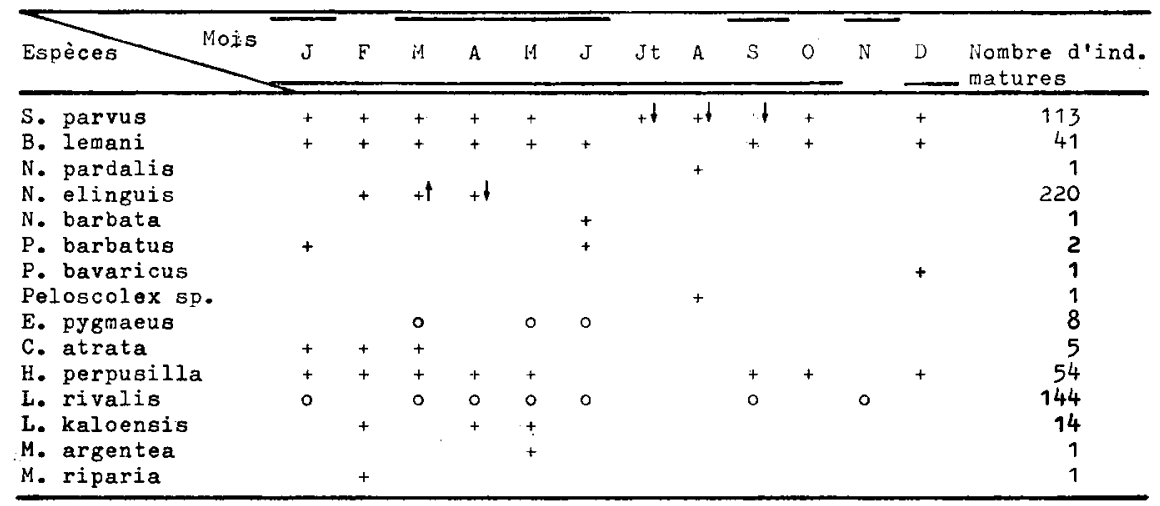

\subsection{Dynamique de la populatica de Nais elinguis dans l'Argens}

Le grand nombre ( 2405$)$ d'individus de $N$. elinguis que nous avons récoltés dans l'Argens nous a permis de suivre son cycle de développement que nous résumons dans la figure 5 .

La période de maturité et la période d'abondance maximale coïncident et se situent à la fin de l'hiver. Le pourcentage d'individus matures par rapport à l'effectif total de la population pendant cette période a été très faible; ceci est en accord avec les observations de Timm (1967 et 1970) et va à l'encontre de celles d'Erseus (1976) et de Learner et al. (1978).

Immédiatement après la période d'abondance maximale (mars), la densité de la population a diminué très brusquement. Un tel phénomène avait été constaté par Learner et al. (1978) dans la rivière Cynon. Ces auteurs proposent comme explication possible de cette diminution le fait déjà souligné par Piguet (1909) et Sperber (1948) que la reproduction asexuée cesserait chez les individus atteignant leur maturité sexuelle. Ce n'est certainement pas le mécanisme qui a déclenché la forte baisse des effectifs de cette espèce dans l'Argens, car même pendant cette période le nombre d'individus matures ne dépassa guère $10 \%$ du total de la population. Les explications qui nous semblent les plus probabı́es dans le cas présent sont: la brusque diminution du taux de scissiparité consécutive au franchissement par la température d'un seuil défavorable, l'apparition massive de prédateurs (larves d'insectes fondamentalement) ou la baisse de la quantité de nourriture disponible. La première et la troisième de ces hypothèses ont déjà 
été vérifiées par Van Cleave (1937) et Poddubnaya (1968) — de façon expérimentale même pour ce dernier auteur.

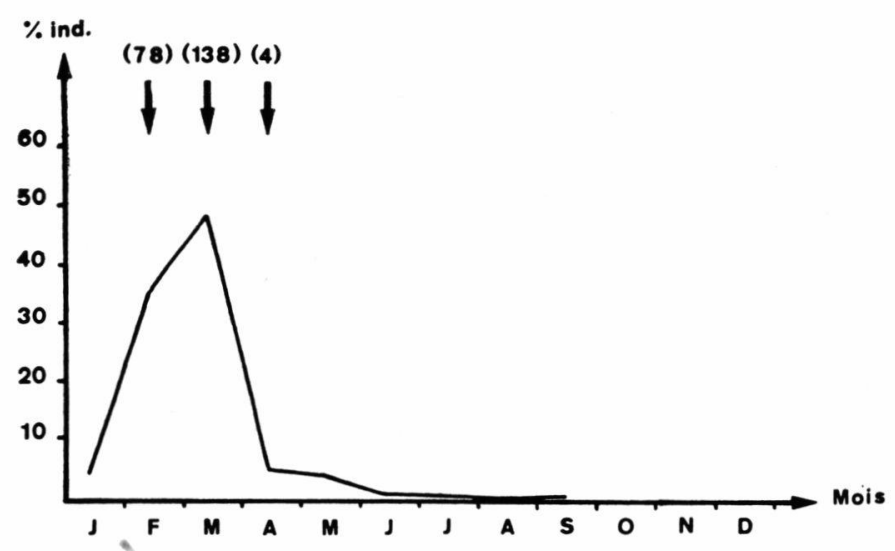

FIg. 5. - Evolution annuelle de la population de Nais elinguis. La flèche indique la présence et le nombre d'individus matures.

Il convient également de signaler que dans d'autres milieux le pic d'abondance maximale et la période de maturité ont été constatés à différentes périodes de l'année: maturité au printemps (Pfannkuche 1979), en été (Piguet et Bretscher 1913 ; Timm 1967 et 1970 ; Erséus 1976), à l'automne (Timm 1967 et 1970); abondance maximale au printemps (Wachs 1967 ; Eyres et al., 1978 ; Pfannkuche 1979), en été (Percival et Whitehead 1930; Timm 1967), en hiver (Ladle 1971). D'autre part, Timm (1967) a observé des périodes de maturité et d'abondance maximale décalées dans le temps au cours de deux années successives, dans une même rivière.

\section{4. - DONNEES ECOLOGIQUES}

\subsection{Autoécologie des principales espèces}

Dans ce paragraphe nous envisagerons les preferendums écologiques manifestés, dans la rivière Argens, par les espèces les plus abondantes (plus de 50 individus récoltés). Les résultats sont résumés dans les tableaux III et VII.

Stylodrilus parvus semble se développer surtout sur les fonds de galets, graviers et sables en courant modéré ou rapide mais elle se rencontre aussi en abondance dans les cailloux en courant rapide. Plus de $40 \%$ des individus ont été récoltés à la station 8 ; cette espèce était aussi assez abondante dans les stations 3 et 9 et elle semble 
仓̀tre absente uniquement des stations 2 et 11 (faciés lénitique) et de celles situées juste en aval de la confluence avec l'Eau Salée (stations 5 et 6). Ces données sont en accord avec celles de Juget (1979) et de Martinez-Ansemil (1981) pour la rivière Tambre (Espagne); mais il convient de souligner que Hrabe (1958) a également rencontré cette espèce en milieu lacustre.

Les preferendums de Bythonomus lemani sont voisins de ceux de l'espèce précédente : fonds de cailloux, galets et graviers souvent légèrement sableux en courant modéré ou rapide. Mais près de $50 \%$ des; individus ont été capturés à la station 5 , première station en aval de la confluence avec l'Eau Salée, d'où $S$. parvus était absente. Nous n'avons pas rencontré $B$. lemani aux stations 4 (lit de mousses et hépatiques en courant rapide à très rapide), 2 et 11 (faciès lénitique). Les observations de Lafont (1977) et celles de Giani (1979) dans plusieurs torrents pyrénéens reflètent le caractère rhéophile de cette espèce dans les cours d'eau. C'est cependant dans les milieux lacustres que cette espèce a été récoltée le plus souvent (Piguet et Bretscher 1913 ; Cernosvitov 1941 ; Juget 1958 ; Juget 1967...). Elle a même été prélevée à $300 \mathrm{~m}$ de profondeur dans le lac Léman par Juget (1958).

Nous n'avons rencontré Ophidonais serpentina que sur les tiges de la végétation palustre qui envahit les stations 2 et 11 . Plusieurs auteurs (Giani 1976; Timm 1970...) signalent un habitat semblable pour cette espèce alors que d'autres (Sperber 1948; Laakso 1967; Juget 1979) l'ont rencontrée sur des fonds vaseux ou sableux; Sarkka (1969) l'a prélevée dans des eaux dont la salinité peut dépasser $3 \%$.

Nais communis est très répandue dans l'Argens. Elle colonise d'avantage les mousses de la station 3 entre lesquelles s'accumule une quantité considérable de matière organique qui provient du secteur situé en amont. Cette espèce se rencontre aussi assez abondamment aux stations 1 (source) et 2. D'après les nombreuses données de la littérature cette espèce est assez ubiquiste et elle a même été souvent rencontrée dans des milieux saumâtres où la salinité atteignait des valeurs de $5 \%$ (Laakso 1969).

Comme $N$. communis, $N$. simplex colonise de préférence les mousses de la station 3 et la végétation de la station 2. A la différence de $N$. communis elle semble absente de la source de l'Argens et des stations situées après la confluence avec l'Eau Salée. Cette espèce a été trouvée aussi bien dans les rivières que dans les lacs par différents auteurs. Piguet et Bretscher (1913) l'ont rencontrée sur des plantes aquatiques et le revêtement des jetées alors que Laakso (1969) l'a prélevée sur des fonds de pierres.

$N$. bretscheri et $N$. barbata se rencontrent de préférence parmi les mousses et les algues filamenteuses en courant lent, modéré ou rapide. La première de ces espèces atteint son développement maximum à la 
station 4 et la deuxième à la station 7 . Aucune des deux n'a été trouvée à la source (station 1) ou dans la station 5 située juste en aval de la confluence avec l'Eau Salée. Selon les données combinées de nombreuses références bibliographiques (Piguet et Bretscher 1913; Juget 1967 ; Sarkka 1968 ; Timm 1970 ; Ladile 1971 ; Lafont 1977 ; Learner et al. $1978 \ldots$..) ces deux espèces ont une large valence écologique en eaux douces mais Laakso (1969) signale également la présence de $N$. barbata dans des milieux où la salinité peut atteindre $5 \%$.

Comme les deux espèces précédentes $N$. pardalis semble se développer surtout parmi les mousses et les algues filamenteuses en courant rapide mais elle est l'espèce dominante de la communauté d'Oligochètes de la source de l'Argens où elle représente près de $50 \%$ des individus. D'après les données de la littérature, cette espèce se rencontre aussi bien dans les rivières que dans les lacs mais contrairement à nos observations elle semble, selon certains auteurs (Wachs 1967; Ladle 1971 ; Martinez-Ansemil 1981..), se développer aussi bien ou même davantage sur les substrats minéraux que dans la végétation aquatique. Laakso (1969) a prélevé cette espèce dans des eaux dont la salinité pouvait dépasser $2 \%$.

$N$. elinguis est de loin l'espèce d'Oligochètes la plus abondante dans l'Argens où elle représente pius de $35 \%$ du total des individus de ce groupe. Nous l'avons récoltée dans tous les biotopes prospectés à l'exception de la végétation en faciès lénitique (stations 2 et 11) et de la source (station 1). Elle semble cependant former des populations plus abondantes sur les fonds de galets et sable en courant modéré ou rapide. D'après les données de la littérature cette espèce apparaît être très eurytope. Elle a été fréquemment trouvée dans les milieux saumâtres dont la salinité peut même atteindre $25 \%$ selon Lasserre (1966);

Nous avons récolté $N$. alpina parmi les mousses et les hépatiques en courant rapide ou très rapide de la station 4 où elle domine la communauté des Oligochètes en compagnie de $N$. elinguis et Lumbricillus kaloensis. Contraitrement à toutes les autres espèces de Nais récoltées dans l'Argens, $N$. alpina semble être uniquement inféodée au milieu lotique. Le caractère rhéophile de cette espèce a été souligné à plusieurs reprises dans la littérature. Quant à la nature du substrat, nos cbservations sont en accord avec celles de Laakso (1969) mais elles vont à l'encontre de celles de Wachs (1967) dans la Fulda et dans l'Isar ; parmi une vaste gamme de substrats cet auteur note une préférence nette de cette espèce pour les fonds caillouteux.

Nous avons récolté Stylaria lacustris dans la végétation en courant très lent ou lent, parmi les algues filamenteuses en courant modéré ou rapide et, plus rarement, sur les fonds de gravier en courant modéré. Nous n'avons prélevé cette espèce que dans les cinq stations situées 
le plus en aval sur la rivière. Ces observations sont en accord avec celles de la littérature. Plusieurs auteurs (Laakso 1967 ; Sarkka 1968 ; Timm 1970...) ont signalé la présence de cette forme dans les milieux saumâtres.

Henlea perpusilla était surtout abondante (plus de $50 \%$ des individus récoltés dans toute la rivière) parmi les mousses de la station 4 en courant rapide ; cette espèce colonise aussi de façon non négligeable les algues filamenteuses des stations 6 et 10 et la végétation de la station 2. Nous ne l'avons pas récoltée à la source (station 1). H. perpusilla se rencontre aussi bien en milieu terrestre (Nielsen et Christensen 1959; Gunst 1965; Nurminen 1970...) que dans les rivières (Kasprzak et Szczesny 1976), les torrents (Giani et Lavandier 1979) où même en milieu saumâtre (Rasmussen 1973). Mais il ne semble pas exister de données bibliographiques sur l'habitat préférentiel de cette espèce en eaux courantes.

TabLEAU VII. - Substrat et vitesse de courant où se développent préférentiellement les principales ( $\mathrm{n}>50$ ) espèces d'Oligochètes de l'Argens. Substrats: A, B, C, G, Gr, H, M, S, T, Vp : même signification que pour les tableaux I et II. Courant: $N$, nul ; $T L$, très lent ; $L$, lent ; $M$, modéré ; $R$, rapide ; $T R$, très rapide. $+, \uparrow, \downarrow:$ même signification que dans le tableau VI.

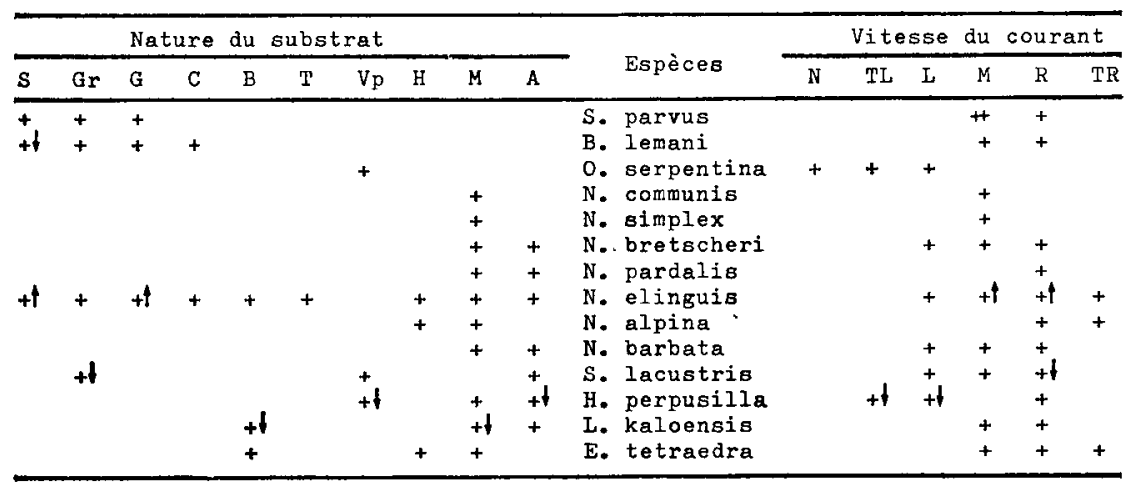

Lumbricillus kaloensis est un Enchytraeidae assez abondant dans la rivière Argens où il semble se développer davantage parmi les algues filamenteuses en courant modéré ou rapide. Nous l'avons rencontré surtout à la station 6 ; il serble être absent de la source et il est très faiblement représenté dans la végétation en faciès lénitique. Mis à part les récoltes de Wachs (1967) dans la rivière Fulda cette espèce n'avait été récoltée que dans le milieu saumâtre (Nielsen et Christensen 1959 : Tynen 1966; Wachs 1967; Kasprzak 1972; Mc Grath 1975 et Healy 1980).

Parmi les nombreux biotopes prospectés Eiseniella tetraedra ne semble absente que dans la végétation en faciès lénitique (stations 1 
(et 2). Elle est surtout abondante dans les mousses et les hépatiques: de la station 4 en courant rapide ou très rapide et sur les fonds pierreux de la station 10. Ces observations sont accord avec hes; nom?breuses données de la bibliographie (Percival et Whitehead 1930;;Benł. nike et Berg 1948 ; Wachs $1967 . .$.$) .$

\subsection{Considérations sur la répartition longitudinale}

La très forte minéralisation des eaux des deux rivières étudièés quii reflète les fortes teneurs en ions $\mathrm{Cl}^{+}, \mathrm{Ca}^{+*}$ et $\mathrm{Mg}^{++}$et qui se traduit par une conductivité très élevée est à I'origine d"ur peuplement en Oli. gochètes un peu particulier pour des eaux contimentales. Ainssi, près: de $40 \%$ des espèces recensées; sont des formzesi quil se rencontnent: aussi bien en eau douce qu'en eau saumâtre.

La brusque augmentation de la tenewr en CI des eaux de- l'Argens après la confluence avec l'Eau Salée emtraîne ame modification dł̆ns; les peuplements d'Oligochètes : certaines espèces, plus caractérïìtiques des milieux saumstrés, telles $N$. elïnguis; et $L$. kaloensis se développent davantage après la confluence alors que d'autres mencontréess plus fréquemment en eaux douces ( $H$. gordioides, $N$. commun $N$. simplex, $N$. bretscheri , $N$. alpina et $H$. perpusilta notamment) forment des populations plus denses en amont de Ia confluence.

Malgré le faible nombre d'individus récoltés dans l'Eau Salée il semblerait, à la vue des tableaux II et IV, que la minéralisation des eaux et la pollution organique par les égoûts du village de Barjols soient aussi à l'origine des différences constatées entre le peuplement du ruisseau des Ecrevisses (station 12) et celui de l'Ea Salée. La présence de Trichodrilus allobrogum dans l'Eau Salée confirme la résistance de cette espèce à certaines pollutions organiques et industrielles comme nous l'avions signalé précédemment (Giani 1979).

Compte tenu de son caractère sténotherme $\left(15^{\circ} \pm 0,5^{\circ} \mathrm{C}\right)$ la source de l'Argens présente un peuplement un peu particulier par rapport aux autres stations de la rivière. En effet ce peuplement est très peu diversifié : seulement 9 espèces sur les 35 recensées y sont représentées. Bien qu'aucune de ces 9 espèces ne soit inféodée à cette station certaines y atteignent leur développement maximum $(\boldsymbol{H}$. gordioides, $N$. pardalis, P. barbatus).

Toutes les différences que nous venons de souligner au niveau de la répartition des espèces dans l'Argens et dans l'Eau Salée, sont, en somme, dues à des changements importants dans les conditions thermiques et surtout chimiques (minéralisation) de l'eau. Cependant comme l'avaient remarqués Wachs (1967) dans les rivières Fulda et Isar (Allemagne), Kasprzak et Szczesny (1976) dans la rivière Raba (Pologne) et Martinez-Ansemil (1981) dans la rivière Tambre (Espa- 
gne), l'influence de ces facteurs - qui sont en fait deux des principaux facteurs qui déterminent la répartition longitudinale d'un grand nombre d'autres groupes d'invertébrés benthiques dans les rivières (Illies et Botosaneanu 1963) - ne se manifeste pas dans l'Argens par un remplacement des différentes populations d'Oligochètes le long du cours axial.

Comme nous l'avions constaté dans le Tambre (Martinez-Ansemil 1981), la répartition longitudinale des populations d'Oligochètes dans l'Argens ne traduit en somme que la distribution à l'intérieur des mosaïques de biotopes définies surtout par le substrat et la vitesse du courant. Ces mosaïques se succèdent de façon très irrégulière le long de l'Argens et ceci explique l'absence d'un remplacement plus ou moins continu d'amont en aval des peuplements d'Oligochètes.

\section{TRAVAUX CITÉS}

Bennike (S. A. B.) et Berg (K.). 1948. - Biological studies on the river Susaa (Oligochaeta). Folia limnol. Scand., $4:$ 40-54.

Berg (K.). 1948. - Biological studies on the river Susaa. Folia limnol. Scand., 4: : $1-318$.

Brinkhurst (R. O.). - Postcript, in Aquatic Oligochaetes Biology. Brinkhurst (R. O.) and Cook (D. G.) éditeurs; Plenum Press, New York, 529 p.

Brinkhurst (R. O.) et JAMIESON (B.G. M.). 1971. - Aquatic Oligochaeta of the World. Oliver and Boyd., Edinburgh, 860 p.

CallleuX (A.). - Limites dimensionnelles et noms des fractions granulométriques.. Bull. Soc. Géol. Fr., $4: 643-646$.

Cernosvitov (L.). 1941. - Oligochaeta from various part of the world. Proc. Zool. Soc. Lond. (B), 111 : 197-236.

Dehzad (B.). 1978. - Etude écologique d'un cours d'eau pollué : l'Eau Salée (Var). Thèse de 3: cycle. Univ. Aix-Marseille III, $147 \mathrm{p}$.

Fomenko (N. V.). 1962. - New species of Oligochaeta for the Dnieper River. Doporid Akad. Nauk. Ukr. R.S.R.. 4 : 542-546.

Frenzel (P.). 1981. - Untersuchungen zur ökologie der Naididae des Bodensees. Die Nische von Chaetogaster und Amphichaeta. Arch. Hydrobiol., 91 : 45-55.

Erseus (C.). 1976. - Littoral Oligochaeta (Annelida) from Eyjafjördur, North. Coast of Iceland. Zool. Scr., 5: 5-11.

Eyres (J. P.), Williams (N. V.) et Pugh-Thomas (M.). 1978. - Ecological studies. on Oligochaeta inhabiting depositing substrata in the Irwell, a polluted. English river. Freshwat. Biol., 8: 25-32.

GianI (N.). 1976. - Les Oligochètes aquatiques du Sud-Ouest de la France. Annls Limnol., 12 : 107-112.

Giani (N.). 1979. - Les Oligochètes aquatiques du Sud-Ouest de la France ( $2^{e}$ note).. Bull. Soc. Hist. Nat. Toulouse, 115 : 347-358.

Giani (N.) et Lavandier (P.). 1977. - Les Oligochètes du torrent d'Estaragne (Pyrénées centrales). Bull. Soc. Hist. Nat. Toulouse, 113 : 234-243.

Gunst (J. H.). 1965. - Enchytraeidae of the Netherlands (Annelida, Oligochaeta).. Beaufortia, 150: 5-12.

Healy (B.). 1980. - Records of Enchytraeidae (Oligochaeta) from Western France: and the Pyrénées. Bull. Mus. Nat. Hist. Nat. Paris, 4 série, $2: 421-443$. 
Hrabe (S.). 1935. - Uber Moraviodrilus pygmaeus n. g., n. sp., Rhyacodrilus falciformis Br., Ilyodrilus bavaricus Oschm. und Bothrioneurum vejdovskyanum St. Spisy vydav. Prir. Fak. Masaryk. Univ., 209: 4-19.

HRABE IS.). 1958. - Die Oligochaeten aus den Seen Dojran und Skadar. Spisy vydav. Prir. Fak. Masaryk. Univ., $397:$ 337-353.

Illies (J.) et Botosaneanu (L.). 1963. - Problèmes et méthodes de la classification et la zonation écologique des eaux courantes, considérées surtout du point de vue faunistique. Mitt. Int. Verein. Theor. Angew. Limnol., 12: 1-57.

Juget (J.). 1958. - Recherches sur la faune de fond du Léman et du lac d'Annecy. Ann. St. Cent. Hydrobiol. appl., 7 : 9-56.

JuGET (J.). 1967. - Quelques données nouvelles sur les Oligochètes du Léman: composition et origine du peuplement. Annls Limnol., 3 : 217-229.

JuGET (J.). 1979. — La texture granulométrique des sédiments et le régime alimentaire des Oligochètes limnicoles. Hydrobiologia, 65 : 145-154.

KASPRZAK (K.). 1972. - Materialy do znajomosci Skaposczetow (Oligochaeta) Wielkopolski. Fragmenta faunistica, $18:$ 99-119.

KASPRZAK (K.) et SzCZESNY (B.). 1976. - Oligochaetes (Oligochaeta) of the river Raba. Acta Hydrobiol., 18 : 75-87.

LaAkso (M.). 1967. - Records of aquatic Oligochaeta from Finland. Ann. Zool. Fenn., 4: 560-566.

LAAKso (M.). 1969 a. - Oligochaeta from brackish water near Tvärminne, SouthWest Finland. Ann. Zool. Fenn., 6 : 98-111.

LaAKSo (M.). 1969 b. - New records of aquatic Oligochaeta from Finland. Antr. Zool. Fenn., $6: 348-352$.

LAdLE (M.). 1971. - The biology of Oligochaeta from Dorset chalk streams. Freswai. Biol., 1 : 83-97.

LaFont (M.). 1977. - Les Oligochètes d'un cours d'eau montagnard pollué : le Bief Rouge. Annls Limnol., 13 : 157-167.

Lasserre (P.). 1966. - Oligochètes marins des côtes de France. I. Bassin d'Arcachon : systématique. Cah. Biol. Mar., $7: 295-317$.

Learner (M. A.), Lochead (G.) et Hughes (B. D.). 1978. - A review of the Biology of British Naididae (Oligochaeta) with emphasis on the lotic environment. Freshwat. Biol., 8 : 357-375.

LEgEZYNSKi (P.). 1971. - Oligochaeta limicola of Wolin Island. Badan. fizjogr. Pol. Zachod. (B), 24: 83-106.

MaRTinez-ANSEMIL (E.). 1978. - Structure horizontale des communautés d'Invertébrés benthiques d'une rivière méditerranéenne, l'Argens (Var). Influence du substrat et du courant. Thèse $3^{*}$ cycle, Univ. Aix-Marseille III, $145 \mathrm{p}$.

Martinez-ANSEMIL (E.). 1981. - Estudio taxonómico y ecológico comparativo de los Oligoquetos de los rios Tambre (Galicia) y Argens (Sur de Francia). Tesis Univ. Santiago de Compostella, 358 p.

MC GRATH (D.). 1975. - Notes on some Irish marine littoral and freshwater Oligochaeta (Annelida). Irish Nat. J., $18:$ 216-218.

Nielsen (C. O.) et Christensen (B.). 1959. - The Enchytraeidae : critical revision and taxonomy of Europaean species. Natura Jutl., 8/9: 1-160.

Nisbet (M.) et Verneaux (J.). 1970. - Composantes chimiques des eaux courantes. Discussion et proposition de classes en tant que bases d'interprétation des analyses chimiques. Annls limnol., $6: 161-190$.

NuRminen (M.). 1970. - Reccrds of Enchytraeidae (Oligochaeta) from the West coast of Greenland. Ann. Zool. Fcn., $7: 199-209$.

Percival (E.) et Whitehead (H.). 1930. - Biological survey of the river Wharfe. II. Report on the invertebrate fauna. J. Ecol., $18: 285-302$. 
Pfannkuche (O.). 1979. - Abundance and life cycle of littoral marine and brackishwater Tubificidae and Naididae (Oligochaeta). In Cyclic phenomena in marine plants and animals. Naylor (E.) et Hartnoll (R. G.) édit., Pergamon Press, Oxford et New York : 103-111.

Piguet (E.). 1909. - Nouvelles observations sur les Naididées. Rev. Suisse Zool., $17: 171-217$.

Piguet (E.) et Bretscher (K.). 1913. - Oligochètes. In : Catalogue des Invertébrés de la Suisse. Mus. Hist. Nat. Genève, 7 : 1-214.

Poddubnaya (T.L.). 1968. - Biology of reproduction of Chaetogaster diaphanus. Trudy Inst. Biol. vnutr. Vod. Akad. Nauk SSSR, 17 : 3-20.

RASMUSSEN (E.). 1973. - Systematics and ecology of the Isefjord marine fauna (Denmark) with a survey of the eelgrass (Zostera) vegetation and its communities. Ophelia, 11: 1-495.

SARKKA (J.). 1968. - Notes on benthic and littoral Oligochaeta in Finland. Ann. Zool. Fenn., 5 : 276-278.

SARKKA (J.). 1969. - The bottom fauna at the mouth of the river Kokemäenjoki, southwestern Finland. Ann. Zool. Fenn., 6: 275-288.

SPERBER (C.). 1948. - A taxonomical study of the Naididae. Zool. Bidr., Upps., 28 : 1-296.

Svetlov (P. G.). 1924. - Einige Angaben ueber die Oligochaetenfauna des Tsherdynbezirkes (Uralgebiet). Bull. Inst. rech. biol. Univ. Perm., 3 : 471-475.

Timm (T.). 1967. - The life cycle of some Oligochaeta. Trudy Karel. otd. Gosniorkh, 5 : 202-204.

Timm (T.). 1970. - On the fauna of the Estonian Oligochaeta. Pedobiologia, 10 : 52-78.

TIMm (T.) et PopchenKo. 1978. - The aquatic Oligochaeta of the Murmansk region. In Hydrobiological researches VII : Season Phenomena in Freshwater Biology. Publication of the Academy of Sciences of the Estonian SSR : 71-132.

TYNen (M. J.). 1966. - A new species of Lumbricillus with a revised check-list of the British Enchytraeidae (Oligochaeta). J. mar. Biol. Ass. U.K., 46: $89-96$.

Van Cleave (C. D.). 1937. - A study of the process of fission in the naid Pristina longiseta. Physiol. Zool., 10 : 299-314.

Vejdovsky (F.). 1891. - Note sur un Tubifex d'Algérie. Mém. Soc. Zool. Fr., 4 : 596-603.

WaCHS (B.). 1967. - Die Oligochaeten-Fauna der Fliebgewässer unter besonderer Berückisichtigung der Beziehungen zwischen der Tubificiden-Bessiedlung und dem Substrat. Arch. Hydrobiol., 63 : 310-386.

Wautier (J.) et Juget (J.). 1969. - La faune benthique et la spéciation dans les lacs subalpins. Bull. Soc. Zool. France, 94 (2) : 277-284. 